\title{
Introduction : Fonctions urbaines et respiration patrimoniale de la ville
}

\section{Nicolas Lebrun}

\section{(2) OpenEdition \\ Journals}

Electronic version

URL: http://journals.openedition.org/rge/5126

DOI: $10.4000 /$ rge. 5126

ISSN: 2108-6478

\section{Publisher}

Association des géographes de l'Est

Printed version

Date of publication: 30 December 2013

ISSN: 0035-3213

\section{Electronic reference}

Nicolas Lebrun, «Introduction: Fonctions urbaines et respiration patrimoniale de la ville », Revue Géographique de l'Est [Online], vol. 53 / 3-4 | 2013, Online since 17 July 2014, connection on 10 December 2020. URL : http://journals.openedition.org/rge/5126 ; DOI : https://doi.org/10.4000/rge. 5126

This text was automatically generated on 10 December 2020.

Tous droits réservés 


\title{
Introduction : Fonctions urbaines et respiration patrimoniale de la ville
}

\author{
Nicolas Lebrun
}

1 Il est courant d'envisager le rôle du patrimoine dans le déploiement d'une thématique fonctionnelle (la qualité patrimoniale comme élément facilitant de la mise en tourisme, par exemple) ou, à l'inverse, le rôle d'une fonction urbaine dans la mise en patrimoine (le commerce comme fonction qui réinvestit certaines des friches industrielles par exemple). L'idée de rapprocher, dans un même numéro de revue, la question des fonctions urbaines et la question patrimoniale, peut dès lors apparaître comme une évidence.

2 Pour autant, les multiples rapprochements entre patrimoine et fonction se font le plus souvent de façon segmentée, le patrimoine étant alors associé à une fonction jugée pertinente : tourisme et patrimoine, commerce et patrimoine, etc. L'idée est donc ici, d'embrasser un ensemble de fonctions urbaines, voire l'articulation entre fonctions urbaines, grâce à la mise en patrimoine de l'espace urbain. Nous espérons que les attitudes, postures et réflexions développées dans ce numéro de la Revue Géographique de l'Est vont permettre de mieux mettre en exergue ces relations.

Pour cela, nous pouvons partir du constat - certainement subjectif - selon lequel le patrimoine semble aujourd'hui être une valeur refuge dans la requalification des espaces urbains.

4 Cet attachement à la valeur patrimoniale du lieu n'a pas toujours été la norme, loin s'en faut. Les logiques fonctionnalistes, en vigueur notamment durant les Trente Glorieuses, sont à l'origine de choix d'urbanisme qui témoignent d'une quasi opposition entre développement fonctionnel et persistance des formes urbaines. Mais à trop vouloir associer forme urbaine et fonctionnalisme, on oublierait peut-être que l'urbanité va bien au-delà de la seule concentration de fonctions urbaines. Il semblait plus facile de créer de nouvelles centralités fonctionnelles que de faire évoluer des centralités existantes jugées obsolètes et inadaptées au Monde moderne. Mais pour autant, il ne s'agissait en aucun cas de nier le besoin de patrimoine: il s'agissait à l'inverse de permettre les conditions d'une "patrimonialisation offensive " (Lebrun, 2006), pour 
laquelle les références renvoient indubitablement à une image de modernité. Créer de nouvelles centralités aptes à devenir les nouveaux points de repère de la ville, innover pour faire patrimoine plutôt que de mettre en patrimoine l'existant.

5 Pour résumer, la création de nouvelles centralités ne s'appuyant pas sur un patrimoine existant repose sur deux constats :

- la valeur symbolique des lieux de pouvoirs associés aux centralités existantes peut être jugée comme datée, surannée ou contraire à l'image de modernité que souhaitent développer les nouveaux acteurs ;

- de fait, les défauts intrinsèques liés à un bâti ancien semblent alors rédhibitoires face à l'exercice d'une activité marquée par de nouveaux concepts empreints de fonctionnalisme.

Mais au fil de la réplication, duplication, standardisation des formes marchandes postfordistes, le message initial s'est brouillé. Les valeurs patrimoniales véhiculées par le fonctionnalisme ont cédé la place à une standardisation facile et irrépressible. C'est le début d'un urbanisme sans âme, très éloigné des valeurs patrimoniales. Ainsi, les années 1970 se sont manifestées par la création de nouvelles centralités périphériques, dans un urbanisme qualifié «d'urbanisme de boîtes " (Péron, 2000) et générateur de «non-lieux» (Augé, 1992) impersonnels.

7 Pour autant, à la faveur des échecs ou des insuffisances des centralités alors créées, le retour aux centralités historiques a pu s'opérer. Comme si la persistance des lieux historiques patrimoniaux était trop forte. Cette persistance des centres est permise par plusieurs éléments :

- les valeurs positives associées à un lieu qui a eu un rôle structurant s'inscrivant dans la durée. Cela permet à ce lieu de bénéficier d'une notoriété confortant sa légitimité, alors que l'image d'une nouvelle centralité reste à construire ;

- la disponibilité fonctionnelle d'un bâti de qualité ;

- la présence d'éléments infrastructurels ayant permis à cet espace de conforter son accessibilité ;

- l'adaptabilité d'une partie du patrimoine immobilier à des usages fonctionnels ;

- le coût de l'émergence d'une polarité créée ex nihilo, une fois l'ensemble des aménités pris en compte.

De fait, nous pourrions dire que chaque type de crise fonctionnelle engendre un rapport à la patrimonialité différent, participant sur le temps long à la pérennité et au renouvellement des centralités fonctionnelles dans la ville: d'abord, une première phase de négation de la centralité existante, comme si l'image patrimoniale du lieu éradiquait toute possibilité de développement fonctionnel; puis une phase de retour à la centralité par le réinvestissement des friches mais toujours en s'appuyant sur une nouvelle fonction urbaine parce que le patrimoine, pour être un levier efficace, doit combiner mémoire du lieu, modernité et renouvellement.

Ainsi, alors même que les différentiels spatiaux sont en perpétuelle recomposition, la rémanence des centres fait figure de garante de la stabilité des repères urbains. L'apparition d'une nouvelle centralité se fait donc du fait de l'insuffisance d'une centralité existante à remplir son rôle. Elle engendre de nouveaux équilibres qui n'entraînent que rarement une disparition des centralités défaillantes. Mais lorsque les nouvelles centralités montreront à leur tour leurs limites, ce sont les centralités originelles qui seront de facto les polarités qui profiteront du changement d'équilibre. 
10 A travers ce numéro, les auteurs vont surtout analyser comment et sous quelle forme l'appel au patrimoine (qu'il s'agisse ou non d'une mise en patrimoine d'élément historiques) permet à la ville d'évoluer sur elle-même dans ses attributs fonctionnels et dans sa symbolique.

11 Géraldine Djament-Tran va ainsi, dans un premier temps, aborder cette évolution des centralités à travers le cas berlinois. Il s'agit de voir en quoi, ce qu'elle qualifie de nouveau cycle patrimonial, lié à la réunification allemande (et donc berlinoise), repose sur une historicité ou une mise en mémoire des lieux urbains. La réunification, événement de politique national et locale, est ici l'élément déclencheur d'un nouveau cycle.

12 Cependant, on pourrait très bien penser que des évolutions structurelles lourdes peuvent elles aussi avoir des répercussions majeures sur le rôle du patrimoine urbain dans la ville. Edith Fagnoni nous présente ainsi en quoi la mondialisation change le rôle du patrimoine du fait de ces propres évolutions. E. Fagnoni parle d'un phénomène de dilatation du patrimoine, impliquant des variations d'échelles spatiales, qui fait que l'appel aux autres échelles que le local n'a jamais été aussi fort dans les préoccupations locales d'aménagement, mais qu'en même temps le besoin de référents identitaires marqués repose sur la différenciation. Entre particularisme et labellisation, et donc reconnaissance selon des standards généralisés, le patrimoine se trouve confronté à la mondialisation dans des rapports complexes.

Qu'il s'agisse d'une mise en articulation avec le Monde ou d'une mise en articulation avec l'Histoire, il s'agit toujours de voir en quoi l'identité patrimoniale s'inscrit dans une dimension mémorielle. C'est cette dimension mémorielle que nous propose d'aborder plus avant Pierre Ginet et Laurène Wiesztort, plus spécifiquement en analysant l'usage politique de cette dimension.

De la politique à la politique urbaine, il n'y a qu'un pas que franchiront ensuite Amélie Nicolas et Laurent Devisme à travers l'analyse du cas nantais. Ils s'intéressent plus particulièrement à la place de la question patrimoniale et des services municipaux dédiés dans la politique urbaine de la métropole nantaise. Cette analyse des jeux d'acteurs permet d'appréhender la complexité de la prise en compte du patrimoine dans l'action publique, alors même que chacun s'accorde à en souligner l'importance.

Les deux articles suivants privilégient l'entrée dans l'action urbaine par la fonction marchande. Marta Alonso Provencio et Antonio Da Cunha s'intéressent d'abord à la question du lien entre espace public et activité commerciale à travers le cas lausannois. Il s'agit de voir en quoi la prise en compte de la qualité urbaine dans un environnement à dominante marchande peut participer au renouvellement urbain et à la durabilité urbaine. Mais aussi d'appréhender en quoi la fonction marchande, peut à l'inverse, du fait de son rôle moteur, participer à la régulation spatiale, notamment par une bonne prise en compte de la question des mobilités, indispensables pour valoriser au mieux les centralités existantes.

16 Anne-Cécile Mermet pour sa part nous présente en quoi la fonction commerciale a été un instrument majeur au service du commerce dans la requalification de centres historiques auparavant dégradés, avec pour conséquence une refonte compète de l'image des dites centralités. Son analyse s'appuie sur deux cas exemplaires : le quartier du Marais à Paris et le Vieux-Lille, tous deux marqués ces dernières années par une 
gentrification marchande très affirmée qui a accompagnée la requalification de l'espace et du patrimoine urbains et la hausse des prix de l'immobilier.

Enfin, Corinne Luxembourg étudie le rapport patrimoine/fonction industrielle dans la ville ;pas seulement pour se poser une fois de plus la question de la reconversion des friches industrielles dans nos espaces urbains, mais bien davantage pour nous monter que la dimension mémorielle des anciens espaces industriels se doit aussi d'être prise en compte dans sa dimension sociale et identitaire pour que le réinvestissement patrimonial puisse se faire au mieux. Il incorpore alors une nécessaire dimension immatérielle garante de l'identité des lieux et des hommes qui ont présidé au développement et à l'histoire de ces espaces fonctionnels.

\section{BIBLIOGRAPHY}

Augé M., 1992, Non-lieux. Introduction à une anthropologie de la surmodernité, Editions du Seuil, Paris Lebrun N., 2006, « Les magasins d'usine : lieux commerciaux ou éléments de valorisation du patrimoine d'espaces urbains en reconversion? », Revue Géographique de l'Est [En ligne], vol. 46 / 3-4.

Péron R., 2000, « Cachez ces boîtes que je ne saurais voir. La recomposition des formes urbaines de la distribution vue par les parlementaires français », Norois, ${ }^{\circ} 185,2000-1$.

\section{AUTHOR}

\section{NICOLAS LEBRUN}

Maître de conférences en géographie - Laboratoire Discontinuités, EA2468 - Université Lille Nord de France, Artois - nicolas.lebrun@univ-artois.fr 